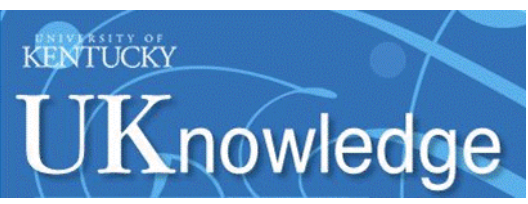

University of Kentucky

UKnowledge

9-2014

\title{
Crystal Structure of 1-methoxy-2,2,2-tris(pyrazol-1-yl)ethane
}

\author{
Ganna Lyubartseva \\ Southern Arkansas University, GannaLyubartseva@saumag.edu \\ Sean Parkin \\ University of Kentucky, s.parkin@uky.edu \\ Morgan D. Coleman \\ Southern Arkansas University \\ Uma Prasad Mallik \\ Southern Arkansas University
}

Follow this and additional works at: https://uknowledge.uky.edu/chemistry_facpub

Part of the Chemistry Commons

Right click to open a feedback form in a new tab to let us know how this document benefits you.

\section{Repository Citation}

Lyubartseva, Ganna; Parkin, Sean; Coleman, Morgan D.; and Mallik, Uma Prasad, "Crystal Structure of 1-methoxy-2,2,2-tris(pyrazol-1-yl)ethane" (2014). Chemistry Faculty Publications. 31.

https://uknowledge.uky.edu/chemistry_facpub/31

This Article is brought to you for free and open access by the Chemistry at UKnowledge. It has been accepted for inclusion in Chemistry Faculty Publications by an authorized administrator of UKnowledge. For more information, please contact UKnowledge@lsv.uky.edu. 


\section{Crystal Structure of 1-methoxy-2,2,2-tris(pyrazol-1-yl)ethane}

\section{Digital Object Identifier (DOI)}

https://doi.org/10.1107/S1600536814018789

\section{Notes/Citation Information}

Published in Acta Crystallographica Section E: Crystallographic Communications, v. 70, part 9, p. o1047-01048.

This is an open-access article distributed under the terms of the Creative Commons Attribution Licence, which permits unrestricted use, distribution, and reproduction in any medium, provided the original authors and source are cited. 


\section{STRUCTURE REPORTS}

ISSN 1600-5368

\section{Crystal structure of 1-methoxy-2,2,2- tris(pyrazol-1-yl)ethane}

\author{
Ganna Lyubartseva, ${ }^{\text {a* Sean Parkin, }}{ }^{\text {b Morgan D. Coleman }}{ }^{\text {a }}$ \\ and Uma Prasad Mallik \\ ${ }^{a}$ Department of Biochemistry and Chemistry, Southern Arkansas University, \\ Magnolia, AR 71753, USA, and 'bepartment of Chemistry, University of Kentucky, \\ Lexington, KY 40506, USA. *Correspondence e-mail: \\ GannaLyubartseva@saumag.edu
}

Received 17 August 2014; accepted 18 August 2014

Edited by H. Stoeckli-Evans, University of Neuchâtel, Switzerland

The title compound, $\mathrm{C}_{12} \mathrm{H}_{14} \mathrm{~N}_{6} \mathrm{O}$, consists of three pyrazole rings bound via nitrogen to the distal ethane carbon of methoxy ethane. The dihedral angles between the three pyrazole rings are $67.62(14), 73.74(14)$, and $78.92(12)^{\circ}$. In the crystal, molecules are linked by bifurcated $\mathrm{C}-\mathrm{H}, \mathrm{H} \cdots \mathrm{N}$ hydrogen bonds, forming double-stranded chains along [001]. The chains are linked via $\mathrm{C}-\mathrm{H} \cdots \mathrm{O}$ hydrogen bonds, forming a three-dimensional framework structure. The crystal was refined as a perfect $(0.5: 0.5)$ inversion twin.

Keywords: crystal structure; tris(pyrazol-1-yl)ethane; scorpionate ligands.

CCDC reference: 1019968

\section{Related literature}

For properties of pyrazole-based tridentate ligands, see: Paulo et al. (2004); Bigmore et al. (2005). For nickel and cobalt complexes of $\mathrm{N}$-donor tridentate scorpionate ligands, see: Lyubartseva et al. $(2011,2012,2013 a, b)$; Lyubartseva \& Parkin (2009). For the synthesis of the title compound, see: Maria et al. (2007).

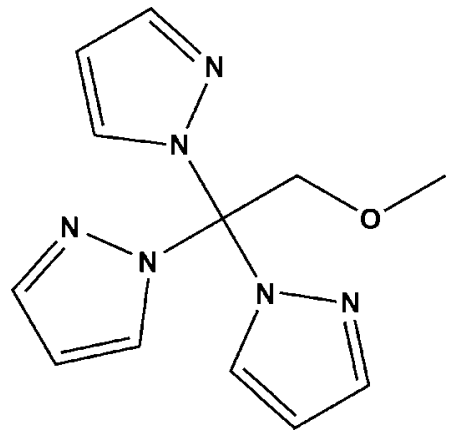

\section{Experimental}

2.1. Crystal data

$\mathrm{C}_{12} \mathrm{H}_{14} \mathrm{~N}_{6} \mathrm{O}$

$M_{r}=258.29$

Monoclinic, $C c$

$a=12.5828$ (3) $\AA$

$b=12.3847$ (3) $\AA$

$c=8.4807$ (2) $\AA$

$\beta=102.5635(11)^{\circ}$

$$
\begin{aligned}
& V=1289.94(5) \AA^{3} \\
& Z=4 \\
& \text { Mo } K \alpha \text { radiation } \\
& \mu=0.09 \mathrm{~mm}^{-1} \\
& T=90 \mathrm{~K} \\
& 0.28 \times 0.20 \times 0.16 \mathrm{~mm}
\end{aligned}
$$

\subsection{Data collection}

Nonius KappaCCD diffractometer Absorption correction: multi-scan (SADABS; Sheldrick, 1996) $T_{\min }=0.749, T_{\max }=0.942$

11397 measured reflections 2934 independent reflections 2386 reflections with $I>2 \sigma(I)$ $R_{\text {int }}=0.032$

\subsection{Refinement}

$R\left[F^{2}>2 \sigma\left(F^{2}\right)\right]=0.042$

$w R\left(F^{2}\right)=0.102$

$S=1.10$

2934 reflections

174 parameters

2 restraints

$\mathrm{H}$-atom parameters constrained

$\Delta \rho_{\max }=0.23 \mathrm{e} \AA^{-3}$

$\Delta \rho_{\min }=-0.18$ e $\AA^{-3}$

Absolute structure: Refined as a perfect (i.e. 50:50) inversion twin

Table 1

Hydrogen-bond geometry $\left(\AA,^{\circ}\right)$.

\begin{tabular}{lllll}
\hline$D-\mathrm{H} \cdots A$ & $D-\mathrm{H}$ & $\mathrm{H} \cdots A$ & $D \cdots A$ & $D-\mathrm{H} \cdots A$ \\
\hline $\mathrm{C} 5-\mathrm{H} 5 A \cdots \mathrm{N} 2^{\mathrm{i}}$ & 0.95 & 2.51 & $3.453(4)$ & 171 \\
$\mathrm{C} 9-\mathrm{H} 9 A \cdots \mathrm{N} 2^{\text {ii }}$ & 0.95 & 2.61 & $3.433(4)$ & 145 \\
$\mathrm{C} 4-\mathrm{H} 4 A \cdots \mathrm{O} 1^{\text {iii }}$ & 0.95 & 2.53 & $3.444(4)$ & 162 \\
\hline
\end{tabular}

Symmetry codes: (i) $x,-y+1, z+\frac{1}{2}$; (ii) $x, y, z+1$; (iii) $x+\frac{1}{2},-y+\frac{1}{2}, z+\frac{1}{2}$.

Data collection: COLLECT (Nonius, 1998); cell refinement: SCALEPACK (Otwinowski \& Minor, 1997); data reduction: DENZO-SMN (Otwinowski \& Minor, 1997); program(s) used to solve structure: SHELXS97 (Sheldrick, 2008); program(s) used to refine structure: SHELXL2014 (Sheldrick, 2008); molecular graphics: $X P$ in SHELXTL (Sheldrick, 2008); software used to prepare material for publication: SHELXL2014 and PLATON (Spek, 2009).

\section{Acknowledgements}

GL is grateful to the Southern Arkansas University Faculty Research Grant for financial support.

Supporting information for this paper is available from the IUCr electronic archives (Reference: SU2774).

\section{References}

Bigmore, H. R., Lawrence, S. C., Mountford, P. \& Tredget, C. S. (2005). Dalton Trans. pp. 635-651.

Lyubartseva, G. \& Parkin, S. (2009). Acta Cryst. E65, m1530.

Lyubartseva, G., Parkin, S. \& Mallik, U. P. (2011). Acta Cryst. E67, m1656$\mathrm{m} 1657$.

Lyubartseva, G., Parkin, S. \& Mallik, U. P. (2013a). Acta Cryst. E69, m532m533.

Lyubartseva, G., Parkin, S. \& Mallik, U. P. (2013b). Acta Cryst. E69, m537. 


\section{data reports}

Lyubartseva, G., Parkin, S., Mallik, U. P. \& Jeon, H. K. (2012). Acta Cryst. E68, m888.

Maria, L., Cunha, S., Videira, M., Gano, L., Paulo, A., Santos, I. C. \& Santos, I. (2007). Dalton Trans. pp. 3010-3019.

Nonius (1998). COLLECT. Nonius BV, Delft, The Netherlands.

Otwinowski, Z. \& Minor, W. (1997). Methods in Enzymology, Vol. 276,

Macromolecular Crystallography, Part A, edited by C. W. Carter Jr \& R. M.

Sweet, pp. 307-326. New York: Academic Press.
Paulo, A., Correia, J. D. G., Campello, M. P. C. \& Santos, I. (2004). Polyhedron, 23, 331-360.

Sheldrick, G. M. (1996). SADABS. University of Göttingen, Germany.

Sheldrick, G. M. (2008). Acta Cryst. A64, 112-122.

Spek, A. L. (2009). Acta Cryst. D65, 148-155. 


\section{supporting information}

Acta Cryst. (2014). E70, o1047-o1048［doi:10.1107/S1600536814018789]

\section{Crystal structure of 1-methoxy-2,2,2-tris(pyrazol-1-yl)ethane}

\section{Ganna Lyubartseva, Sean Parkin, Morgan D. Coleman and Uma Prasad Mallik}

\section{S1. Synthesis and crystallization}

The title compound was prepared using the published procedure (Maria et al., 2007). Colourless block-like crystals were obtained by slow evaporation of a diethyl ether solution of pure product. Spectral and other characterizations are in good accordance with the previously reported data (Maria et al., 2007).

\section{S2. Refinement}

$\mathrm{H}$ atoms were located in difference Fourier maps, but were subsequently included in the refinement using a riding model approximation: $\mathrm{C}-\mathrm{H}=0.95-0.99 \AA$ with $\mathrm{U}_{\mathrm{iso}}(\mathrm{H})=1.5 U_{\mathrm{eq}}(\mathrm{C}$-methyl $)$ and $=1.2 U_{\mathrm{eq}}(\mathrm{C})$ for other $\mathrm{H}$ atoms. The crystal was refined as a perfect $(0.5: 0.5)$ inversion twin.

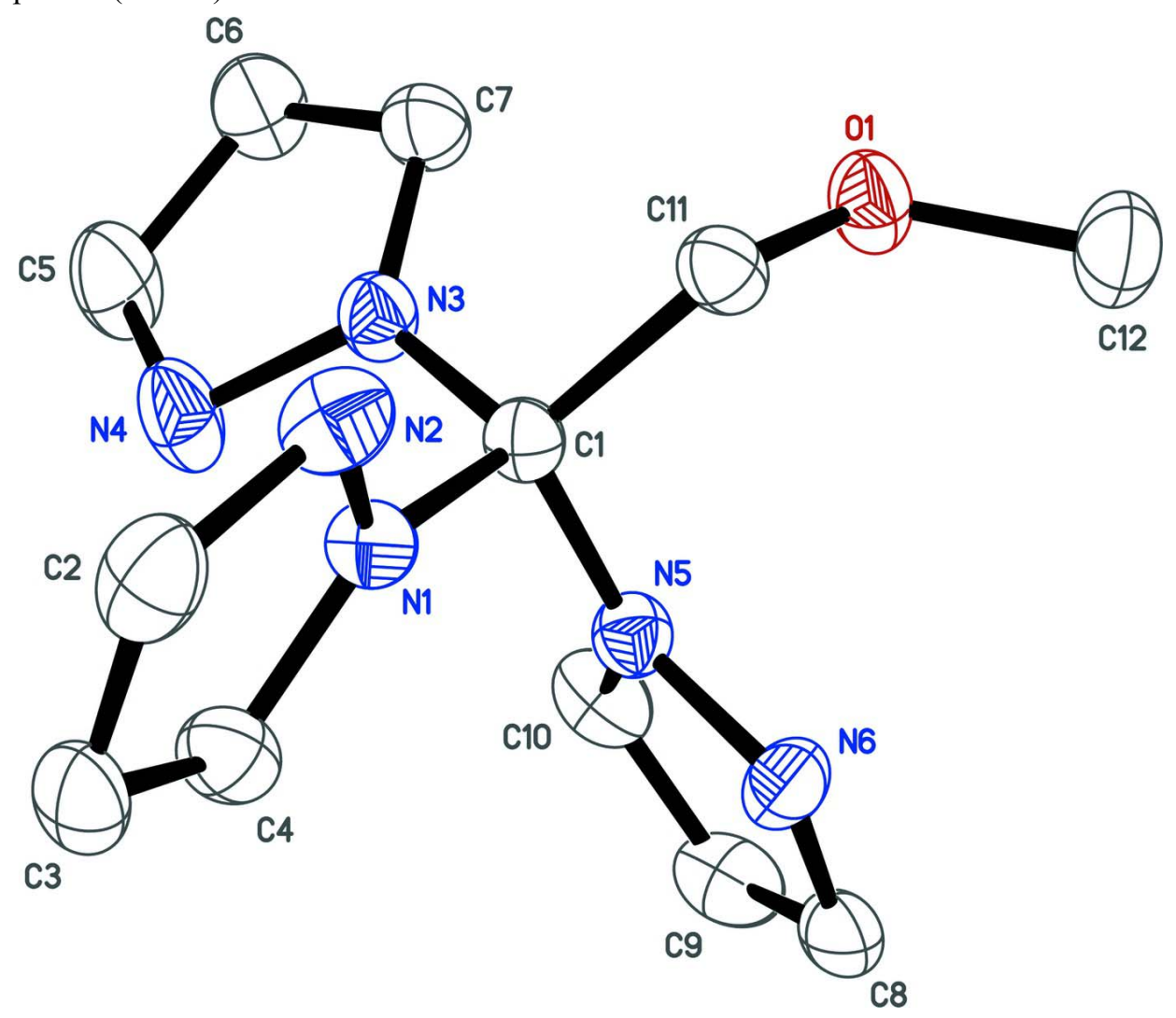

Figure 1

View of molecular structure of the title molecule, with atom labelling. Displacement ellipsoids are drawn at the 50\% probability level. $\mathrm{H}$ atoms have been omitted for clarity. 


\section{1-Methoxy-2,2,2-tris(pyrazol-1-yl)ethane}

Crystal data

$\mathrm{C}_{12} \mathrm{H}_{14} \mathrm{~N}_{6} \mathrm{O}$

$M_{r}=258.29$

Monoclinic, $C c$

$a=12.5828(3) \AA$

$b=12.3847$ (3) $\AA$

$c=8.4807(2) \AA$

$\beta=102.5635(11)^{\circ}$

$V=1289.94(5) \AA^{3}$

$Z=4$

Data collection

Nonius KappaCCD diffractometer

Radiation source: fine-focus sealed-tube

Detector resolution: 9.1 pixels $\mathrm{mm}^{-1}$

$\varphi$ and $\omega$ scans at fixed $\chi=55^{\circ}$

Absorption correction: multi-scan

(SADABS; Sheldrick, 1996)

$T_{\min }=0.749, T_{\max }=0.942$

Refinement

Refinement on $F^{2}$

Least-squares matrix: full

$R\left[F^{2}>2 \sigma\left(F^{2}\right)\right]=0.042$

$w R\left(F^{2}\right)=0.102$

$S=1.10$

2934 reflections

174 parameters

2 restraints

Primary atom site location: structure-invariant direct methods

Secondary atom site location: difference Fourier map

Special details

Experimental. The crystal was mounted with polyisobutene oil on the tip of a fine glass fibre, which was fastened in a copper mounting pin with electrical solder. It was placed directly into the cold gas stream of a liquid nitrogen based cryostat, according to published methods (Hope, 1994; Parkin \& Hope, 1998).

Diffraction data were collected with the crystal at $90 \mathrm{~K}$, which is standard practice in this laboratory for the majority of flash-cooled crystals.

Geometry. All e.s.d.'s (except the e.s.d. in the dihedral angle between two l.s. planes) are estimated using the full covariance matrix. The cell e.s.d.'s are taken into account individually in the estimation of e.s.d.'s in distances, angles and torsion angles; correlations between e.s.d.'s in cell parameters are only used when they are defined by crystal symmetry. An approximate (isotropic) treatment of cell e.s.d.'s is used for estimating e.s.d.'s involving 1.s. planes.

Refinement. Refinement progress was checked using PLATON (Spek, 2009) and by an $R$-tensor (Parkin, 2000). The final model was further checked with the IUCr utility checkCIF.

Fractional atomic coordinates and isotropic or equivalent isotropic displacement parameters $\left(\AA^{2}\right)$

\begin{tabular}{lllll}
\hline & $x$ & $y$ & $z$ & $U_{\text {iso }} / U_{\text {eq }}$ \\
\hline O1 & $0.34875(17)$ & $0.16856(16)$ & $0.5089(3)$ & $0.0305(5)$
\end{tabular}

Acta Cryst. (2014). E70, 01047-01048

sup-2 


\begin{tabular}{|c|c|c|c|c|}
\hline N1 & 0.62903 (19) & $0.25690(19)$ & $0.5191(3)$ & $0.0229(5)$ \\
\hline $\mathrm{N} 2$ & $0.6299(2)$ & $0.2508(2)$ & $0.3598(3)$ & $0.0291(6)$ \\
\hline N3 & $0.4892(2)$ & $0.35743(18)$ & $0.5996(3)$ & $0.0255(6)$ \\
\hline N4 & $0.5617(2)$ & $0.4320(2)$ & $0.6770(4)$ & $0.0378(7)$ \\
\hline N5 & $0.5520(2)$ & $0.1924(2)$ & $0.7270(3)$ & $0.0236(5)$ \\
\hline N6 & $0.5898(2)$ & $0.0891(2)$ & $0.7266(3)$ & $0.0296(6)$ \\
\hline $\mathrm{C} 1$ & $0.5280(2)$ & $0.2497(2)$ & $0.5740(3)$ & $0.0212(6)$ \\
\hline $\mathrm{C} 2$ & $0.7343(3)$ & $0.2658(3)$ & $0.3557(4)$ & $0.0336(7)$ \\
\hline $\mathrm{H} 2 \mathrm{~A}$ & 0.7610 & 0.2658 & 0.2591 & $0.040^{*}$ \\
\hline $\mathrm{C} 3$ & $0.8001(2)$ & $0.2816(2)$ & $0.5100(4)$ & $0.0318(7)$ \\
\hline $\mathrm{H} 3 \mathrm{~A}$ & 0.8765 & 0.2933 & 0.5376 & $0.038^{*}$ \\
\hline $\mathrm{C} 4$ & $0.7295(3)$ & $0.2762(2)$ & $0.6118(4)$ & $0.0303(7)$ \\
\hline $\mathrm{H} 4 \mathrm{~A}$ & 0.7475 & 0.2845 & 0.7259 & $0.036^{*}$ \\
\hline $\mathrm{C} 5$ & $0.5010(3)$ & $0.5170(3)$ & $0.6921(5)$ & $0.0426(9)$ \\
\hline $\mathrm{H} 5 \mathrm{~A}$ & 0.5290 & 0.5831 & 0.7406 & $0.051 *$ \\
\hline C6 & $0.3900(3)$ & $0.4977(3)$ & $0.6277(5)$ & $0.0394(8)$ \\
\hline H6A & 0.3311 & 0.5461 & 0.6252 & $0.047 *$ \\
\hline $\mathrm{C} 7$ & $0.3848(3)$ & $0.3954(3)$ & $0.5699(4)$ & $0.0307(7)$ \\
\hline H7A & 0.3210 & 0.3574 & 0.5187 & $0.037^{*}$ \\
\hline $\mathrm{C} 8$ & $0.6077(3)$ & $0.0592(3)$ & $0.8804(4)$ & $0.0330(7)$ \\
\hline H8A & 0.6344 & -0.0098 & 0.9187 & $0.040 *$ \\
\hline C9 & $0.5825(3)$ & $0.1410(3)$ & $0.9791(4)$ & $0.0379(8)$ \\
\hline H9A & 0.5884 & 0.1385 & 1.0927 & $0.045^{*}$ \\
\hline $\mathrm{C} 10$ & $0.5474(2)$ & $0.2258(3)$ & $0.8775(4)$ & $0.0324(7)$ \\
\hline H10A & 0.5244 & 0.2946 & 0.9067 & $0.039 *$ \\
\hline C11 & $0.4437(2)$ & $0.1869(2)$ & $0.4498(4)$ & $0.0252(6)$ \\
\hline H11A & 0.4249 & 0.2284 & 0.3478 & $0.030^{*}$ \\
\hline H11B & 0.4751 & 0.1169 & 0.4266 & $0.030^{*}$ \\
\hline $\mathrm{C} 12$ & $0.3184(3)$ & 0.0580 & $0.5101(5)$ & $0.0384(8)$ \\
\hline H12A & 0.2517 & 0.0515 & 0.5511 & $0.058^{*}$ \\
\hline H12B & 0.3770 & 0.0171 & 0.5800 & $0.058^{*}$ \\
\hline $\mathrm{H} 12 \mathrm{C}$ & 0.3057 & 0.0291 & 0.4000 & $0.058^{*}$ \\
\hline
\end{tabular}

Atomic displacement parameters $\left(\AA^{2}\right)$

\begin{tabular}{lllllll}
\hline & $U^{11}$ & $U^{22}$ & $U^{33}$ & $U^{12}$ & $U^{13}$ & $U^{23}$ \\
\hline O1 & $0.0238(11)$ & $0.0263(11)$ & $0.0426(13)$ & $-0.0028(9)$ & $0.0099(9)$ & $-0.0036(9)$ \\
N1 & $0.0226(13)$ & $0.0254(12)$ & $0.0214(13)$ & $0.0009(10)$ & $0.0062(10)$ & $0.0004(10)$ \\
N2 & $0.0335(15)$ & $0.0325(14)$ & $0.0239(13)$ & $0.0045(11)$ & $0.0115(11)$ & $0.0029(11)$ \\
N3 & $0.0233(12)$ & $0.0213(12)$ & $0.0324(13)$ & $-0.0003(10)$ & $0.0072(10)$ & $-0.0024(11)$ \\
N4 & $0.0282(14)$ & $0.0256(14)$ & $0.0600(19)$ & $-0.0051(12)$ & $0.0103(13)$ & $-0.0140(13)$ \\
N5 & $0.0247(12)$ & $0.0237(12)$ & $0.0227(12)$ & $-0.0018(10)$ & $0.0058(10)$ & $-0.0001(10)$ \\
N6 & $0.0359(15)$ & $0.0213(13)$ & $0.0294(14)$ & $0.0003(10)$ & $0.0025(11)$ & $0.0033(10)$ \\
C1 & $0.0197(14)$ & $0.0219(14)$ & $0.0233(15)$ & $0.0004(10)$ & $0.0074(12)$ & $-0.0010(11)$ \\
C2 & $0.0357(17)$ & $0.0306(17)$ & $0.0400(19)$ & $0.0029(14)$ & $0.0203(15)$ & $0.0051(14)$ \\
C3 & $0.0222(15)$ & $0.0285(15)$ & $0.048(2)$ & $0.0000(12)$ & $0.0143(15)$ & $0.0000(14)$ \\
C4 & $0.0257(16)$ & $0.0316(17)$ & $0.0334(17)$ & $0.0020(13)$ & $0.0057(13)$ & $-0.0024(13)$ \\
C5 & $0.0370(18)$ & $0.0272(18)$ & $0.064(2)$ & $-0.0032(14)$ & $0.0130(17)$ & $-0.0149(16)$
\end{tabular}


supporting information

\begin{tabular}{lllllll} 
C6 & $0.0308(17)$ & $0.0274(17)$ & $0.061(2)$ & $0.0062(14)$ & $0.0116(16)$ & $-0.0077(15)$ \\
C7 & $0.0249(15)$ & $0.0287(16)$ & $0.0374(17)$ & $0.0024(12)$ & $0.0042(13)$ & $-0.0008(13)$ \\
C8 & $0.0248(16)$ & $0.0364(18)$ & $0.0353(18)$ & $-0.0083(14)$ & $0.0009(13)$ & $0.0113(15)$ \\
C9 & $0.0300(17)$ & $0.060(2)$ & $0.0246(16)$ & $-0.0035(16)$ & $0.0075(13)$ & $0.0056(15)$ \\
C10 & $0.0260(16)$ & $0.0457(19)$ & $0.0282(16)$ & $-0.0028(14)$ & $0.0122(13)$ & $-0.0058(14)$ \\
C11 & $0.0214(15)$ & $0.0256(14)$ & $0.0280(15)$ & $0.0008(11)$ & $0.0041(12)$ & $-0.0027(12)$ \\
C12 & $0.038(2)$ & $0.0282(17)$ & $0.051(2)$ & $-0.0059(14)$ & $0.0126(17)$ & $0.0031(16)$ \\
\hline
\end{tabular}

Geometric parameters $\left(\AA,{ }^{\circ}\right)$

\begin{tabular}{|c|c|c|c|}
\hline $\mathrm{O} 1-\mathrm{C} 11$ & $1.411(3)$ & $\mathrm{C} 3-\mathrm{H} 3 \mathrm{~A}$ & 0.9500 \\
\hline $\mathrm{O} 1-\mathrm{C} 12$ & $1.423(4)$ & $\mathrm{C} 4-\mathrm{H} 4 \mathrm{~A}$ & 0.9500 \\
\hline $\mathrm{N} 1-\mathrm{N} 2$ & $1.356(3)$ & $\mathrm{C} 5-\mathrm{C} 6$ & $1.405(5)$ \\
\hline $\mathrm{N} 1-\mathrm{C} 4$ & $1.357(4)$ & $\mathrm{C} 5-\mathrm{H} 5 \mathrm{~A}$ & 0.9500 \\
\hline $\mathrm{N} 1-\mathrm{C} 1$ & $1.448(3)$ & $\mathrm{C} 6-\mathrm{C} 7$ & $1.355(5)$ \\
\hline $\mathrm{N} 2-\mathrm{C} 2$ & $1.335(4)$ & $\mathrm{C} 6-\mathrm{H} 6 \mathrm{~A}$ & 0.9500 \\
\hline $\mathrm{N} 3-\mathrm{N} 4$ & $1.363(4)$ & $\mathrm{C} 7-\mathrm{H} 7 \mathrm{~A}$ & 0.9500 \\
\hline N3-C7 & $1.365(4)$ & $\mathrm{C} 8-\mathrm{C} 9$ & $1.394(5)$ \\
\hline $\mathrm{N} 3-\mathrm{C} 1$ & $1.454(3)$ & $\mathrm{C} 8-\mathrm{H} 8 \mathrm{~A}$ & 0.9500 \\
\hline $\mathrm{N} 4-\mathrm{C} 5$ & $1.323(4)$ & $\mathrm{C} 9-\mathrm{C} 10$ & $1.369(5)$ \\
\hline $\mathrm{N} 5-\mathrm{C} 10$ & $1.354(4)$ & $\mathrm{C} 9-\mathrm{H} 9 \mathrm{~A}$ & 0.9500 \\
\hline N5-N6 & $1.364(3)$ & $\mathrm{C} 10-\mathrm{H} 10 \mathrm{~A}$ & 0.9500 \\
\hline $\mathrm{N} 5-\mathrm{C} 1$ & $1.452(4)$ & $\mathrm{C} 11-\mathrm{H} 11 \mathrm{~A}$ & 0.9900 \\
\hline $\mathrm{N} 6-\mathrm{C} 8$ & $1.327(4)$ & C11-H11B & 0.9900 \\
\hline $\mathrm{C} 1-\mathrm{C} 11$ & $1.534(4)$ & $\mathrm{C} 12-\mathrm{H} 12 \mathrm{~A}$ & 0.9800 \\
\hline $\mathrm{C} 2-\mathrm{C} 3$ & $1.402(5)$ & $\mathrm{C} 12-\mathrm{H} 12 \mathrm{~B}$ & 0.9800 \\
\hline $\mathrm{C} 2-\mathrm{H} 2 \mathrm{~A}$ & 0.9500 & $\mathrm{C} 12-\mathrm{H} 12 \mathrm{C}$ & 0.9800 \\
\hline $\mathrm{C} 3-\mathrm{C} 4$ & $1.369(4)$ & & \\
\hline $\mathrm{C} 11-\mathrm{O} 1-\mathrm{C} 12$ & $114.0(2)$ & $\mathrm{N} 4-\mathrm{C} 5-\mathrm{H} 5 \mathrm{~A}$ & 124.0 \\
\hline $\mathrm{N} 2-\mathrm{N} 1-\mathrm{C} 4$ & $112.3(2)$ & $\mathrm{C} 6-\mathrm{C} 5-\mathrm{H} 5 \mathrm{~A}$ & 124.0 \\
\hline $\mathrm{N} 2-\mathrm{N} 1-\mathrm{C} 1$ & $121.0(2)$ & $\mathrm{C} 7-\mathrm{C} 6-\mathrm{C} 5$ & $105.3(3)$ \\
\hline $\mathrm{C} 4-\mathrm{N} 1-\mathrm{C} 1$ & $126.7(2)$ & $\mathrm{C} 7-\mathrm{C} 6-\mathrm{H} 6 \mathrm{~A}$ & 127.3 \\
\hline $\mathrm{C} 2-\mathrm{N} 2-\mathrm{N} 1$ & $103.8(3)$ & $\mathrm{C} 5-\mathrm{C} 6-\mathrm{H} 6 \mathrm{~A}$ & 127.3 \\
\hline $\mathrm{N} 4-\mathrm{N} 3-\mathrm{C} 7$ & $111.9(2)$ & $\mathrm{C} 6-\mathrm{C} 7-\mathrm{N} 3$ & $106.7(3)$ \\
\hline $\mathrm{N} 4-\mathrm{N} 3-\mathrm{C} 1$ & $118.8(2)$ & $\mathrm{C} 6-\mathrm{C} 7-\mathrm{H} 7 \mathrm{~A}$ & 126.7 \\
\hline $\mathrm{C} 7-\mathrm{N} 3-\mathrm{C} 1$ & $129.0(2)$ & $\mathrm{N} 3-\mathrm{C} 7-\mathrm{H} 7 \mathrm{~A}$ & 126.7 \\
\hline $\mathrm{C} 5-\mathrm{N} 4-\mathrm{N} 3$ & $104.2(3)$ & $\mathrm{N} 6-\mathrm{C} 8-\mathrm{C} 9$ & $112.0(3)$ \\
\hline $\mathrm{C} 10-\mathrm{N} 5-\mathrm{N} 6$ & $112.0(3)$ & $\mathrm{N} 6-\mathrm{C} 8-\mathrm{H} 8 \mathrm{~A}$ & 124.0 \\
\hline $\mathrm{C} 10-\mathrm{N} 5-\mathrm{C} 1$ & $130.5(3)$ & $\mathrm{C} 9-\mathrm{C} 8-\mathrm{H} 8 \mathrm{~A}$ & 124.0 \\
\hline $\mathrm{N} 6-\mathrm{N} 5-\mathrm{C} 1$ & $117.4(2)$ & $\mathrm{C} 10-\mathrm{C} 9-\mathrm{C} 8$ & $105.3(3)$ \\
\hline $\mathrm{C} 8-\mathrm{N} 6-\mathrm{N} 5$ & $104.1(3)$ & $\mathrm{C} 10-\mathrm{C} 9-\mathrm{H} 9 \mathrm{~A}$ & 127.4 \\
\hline $\mathrm{N} 1-\mathrm{C} 1-\mathrm{N} 5$ & $106.9(2)$ & $\mathrm{C} 8-\mathrm{C} 9-\mathrm{H} 9 \mathrm{~A}$ & 127.4 \\
\hline $\mathrm{N} 1-\mathrm{C} 1-\mathrm{N} 3$ & $109.8(2)$ & $\mathrm{N} 5-\mathrm{C} 10-\mathrm{C} 9$ & $106.6(3)$ \\
\hline $\mathrm{N} 5-\mathrm{C} 1-\mathrm{N} 3$ & $109.0(2)$ & $\mathrm{N} 5-\mathrm{C} 10-\mathrm{H} 10 \mathrm{~A}$ & 126.7 \\
\hline $\mathrm{N} 1-\mathrm{C} 1-\mathrm{C} 11$ & $109.6(2)$ & $\mathrm{C} 9-\mathrm{C} 10-\mathrm{H} 10 \mathrm{~A}$ & 126.7 \\
\hline $\mathrm{N} 5-\mathrm{C} 1-\mathrm{C} 11$ & $110.1(2)$ & $\mathrm{O} 1-\mathrm{C} 11-\mathrm{C} 1$ & $110.5(2)$ \\
\hline $\mathrm{N} 3-\mathrm{C} 1-\mathrm{C} 11$ & $111.3(2)$ & $\mathrm{O} 1-\mathrm{C} 11-\mathrm{H} 11 \mathrm{~A}$ & 109.5 \\
\hline
\end{tabular}




\begin{tabular}{|c|c|c|c|}
\hline $\mathrm{N} 2-\mathrm{C} 2-\mathrm{C} 3$ & $112.3(3)$ & $\mathrm{C} 1-\mathrm{C} 11-\mathrm{H} 11 \mathrm{~A}$ & 109.5 \\
\hline $\mathrm{N} 2-\mathrm{C} 2-\mathrm{H} 2 \mathrm{~A}$ & 123.8 & $\mathrm{O} 1-\mathrm{C} 11-\mathrm{H} 11 \mathrm{~B}$ & 109.5 \\
\hline $\mathrm{C} 3-\mathrm{C} 2-\mathrm{H} 2 \mathrm{~A}$ & 123.8 & $\mathrm{C} 1-\mathrm{C} 11-\mathrm{H} 11 \mathrm{~B}$ & 109.5 \\
\hline $\mathrm{C} 4-\mathrm{C} 3-\mathrm{C} 2$ & $104.4(3)$ & $\mathrm{H} 11 \mathrm{~A}-\mathrm{C} 11-\mathrm{H} 11 \mathrm{~B}$ & 108.1 \\
\hline $\mathrm{C} 4-\mathrm{C} 3-\mathrm{H} 3 \mathrm{~A}$ & 127.8 & $\mathrm{O} 1-\mathrm{C} 12-\mathrm{H} 12 \mathrm{~A}$ & 109.5 \\
\hline $\mathrm{C} 2-\mathrm{C} 3-\mathrm{H} 3 \mathrm{~A}$ & 127.8 & $\mathrm{O} 1-\mathrm{C} 12-\mathrm{H} 12 \mathrm{~B}$ & 109.5 \\
\hline $\mathrm{N} 1-\mathrm{C} 4-\mathrm{C} 3$ & $107.1(3)$ & $\mathrm{H} 12 \mathrm{~A}-\mathrm{C} 12-\mathrm{H} 12 \mathrm{~B}$ & 109.5 \\
\hline $\mathrm{N} 1-\mathrm{C} 4-\mathrm{H} 4 \mathrm{~A}$ & 126.4 & $\mathrm{O} 1-\mathrm{C} 12-\mathrm{H} 12 \mathrm{C}$ & 109.5 \\
\hline $\mathrm{C} 3-\mathrm{C} 4-\mathrm{H} 4 \mathrm{~A}$ & 126.4 & $\mathrm{H} 12 \mathrm{~A}-\mathrm{C} 12-\mathrm{H} 12 \mathrm{C}$ & 109.5 \\
\hline $\mathrm{N} 4-\mathrm{C} 5-\mathrm{C} 6$ & $112.0(3)$ & $\mathrm{H} 12 \mathrm{~B}-\mathrm{C} 12-\mathrm{H} 12 \mathrm{C}$ & 109.5 \\
\hline $\mathrm{C} 4-\mathrm{N} 1-\mathrm{N} 2-\mathrm{C} 2$ & $0.6(3)$ & $\mathrm{N} 4-\mathrm{N} 3-\mathrm{C} 1-\mathrm{C} 11$ & $-165.4(3)$ \\
\hline $\mathrm{C} 1-\mathrm{N} 1-\mathrm{N} 2-\mathrm{C} 2$ & $177.0(3)$ & $\mathrm{C} 7-\mathrm{N} 3-\mathrm{C} 1-\mathrm{C} 11$ & $21.3(4)$ \\
\hline $\mathrm{C} 7-\mathrm{N} 3-\mathrm{N} 4-\mathrm{C} 5$ & $-1.0(4)$ & $\mathrm{N} 1-\mathrm{N} 2-\mathrm{C} 2-\mathrm{C} 3$ & $-0.1(3)$ \\
\hline $\mathrm{C} 1-\mathrm{N} 3-\mathrm{N} 4-\mathrm{C} 5$ & $-175.4(3)$ & $\mathrm{N} 2-\mathrm{C} 2-\mathrm{C} 3-\mathrm{C} 4$ & $-0.4(4)$ \\
\hline $\mathrm{C} 10-\mathrm{N} 5-\mathrm{N} 6-\mathrm{C} 8$ & $0.5(3)$ & $\mathrm{N} 2-\mathrm{N} 1-\mathrm{C} 4-\mathrm{C} 3$ & $-0.9(3)$ \\
\hline $\mathrm{C} 1-\mathrm{N} 5-\mathrm{N} 6-\mathrm{C} 8$ & $178.0(2)$ & $\mathrm{C} 1-\mathrm{N} 1-\mathrm{C} 4-\mathrm{C} 3$ & $-177.1(3)$ \\
\hline $\mathrm{N} 2-\mathrm{N} 1-\mathrm{C} 1-\mathrm{N} 5$ & $144.6(2)$ & $\mathrm{C} 2-\mathrm{C} 3-\mathrm{C} 4-\mathrm{N} 1$ & $0.8(3)$ \\
\hline $\mathrm{C} 4-\mathrm{N} 1-\mathrm{C} 1-\mathrm{N} 5$ & $-39.6(4)$ & $\mathrm{N} 3-\mathrm{N} 4-\mathrm{C} 5-\mathrm{C} 6$ & $0.9(4)$ \\
\hline $\mathrm{N} 2-\mathrm{N} 1-\mathrm{C} 1-\mathrm{N} 3$ & $-97.3(3)$ & $\mathrm{N} 4-\mathrm{C} 5-\mathrm{C} 6-\mathrm{C} 7$ & $-0.5(5)$ \\
\hline $\mathrm{C} 4-\mathrm{N} 1-\mathrm{C} 1-\mathrm{N} 3$ & $78.6(3)$ & $\mathrm{C} 5-\mathrm{C} 6-\mathrm{C} 7-\mathrm{N} 3$ & $-0.2(4)$ \\
\hline $\mathrm{N} 2-\mathrm{N} 1-\mathrm{C} 1-\mathrm{C} 11$ & $25.2(3)$ & $\mathrm{N} 4-\mathrm{N} 3-\mathrm{C} 7-\mathrm{C} 6$ & $0.8(4)$ \\
\hline $\mathrm{C} 4-\mathrm{N} 1-\mathrm{C} 1-\mathrm{C} 11$ & $-158.9(3)$ & $\mathrm{C} 1-\mathrm{N} 3-\mathrm{C} 7-\mathrm{C} 6$ & $174.4(3)$ \\
\hline $\mathrm{C} 10-\mathrm{N} 5-\mathrm{C} 1-\mathrm{N} 1$ & $115.6(3)$ & $\mathrm{N} 5-\mathrm{N} 6-\mathrm{C} 8-\mathrm{C} 9$ & $-0.2(3)$ \\
\hline $\mathrm{N} 6-\mathrm{N} 5-\mathrm{C} 1-\mathrm{N} 1$ & $-61.3(3)$ & $\mathrm{N} 6-\mathrm{C} 8-\mathrm{C} 9-\mathrm{C} 10$ & $-0.2(4)$ \\
\hline $\mathrm{C} 10-\mathrm{N} 5-\mathrm{C} 1-\mathrm{N} 3$ & $-3.0(4)$ & $\mathrm{N} 6-\mathrm{N} 5-\mathrm{C} 10-\mathrm{C} 9$ & $-0.6(3)$ \\
\hline $\mathrm{N} 6-\mathrm{N} 5-\mathrm{C} 1-\mathrm{N} 3$ & $-179.9(2)$ & $\mathrm{C} 1-\mathrm{N} 5-\mathrm{C} 10-\mathrm{C} 9$ & $-177.7(3)$ \\
\hline $\mathrm{C} 10-\mathrm{N} 5-\mathrm{C} 1-\mathrm{C} 11$ & $-125.4(3)$ & $\mathrm{C} 8-\mathrm{C} 9-\mathrm{C} 10-\mathrm{N} 5$ & $0.4(3)$ \\
\hline $\mathrm{N} 6-\mathrm{N} 5-\mathrm{C} 1-\mathrm{C} 11$ & $57.7(3)$ & $\mathrm{C} 12-\mathrm{O} 1-\mathrm{C} 11-\mathrm{C} 1$ & $-124.7(3)$ \\
\hline $\mathrm{N} 4-\mathrm{N} 3-\mathrm{C} 1-\mathrm{N} 1$ & $-43.9(3)$ & $\mathrm{N} 1-\mathrm{C} 1-\mathrm{C} 11-\mathrm{O} 1$ & $173.5(2)$ \\
\hline $\mathrm{C} 7-\mathrm{N} 3-\mathrm{C} 1-\mathrm{N} 1$ & $142.8(3)$ & $\mathrm{N} 5-\mathrm{C} 1-\mathrm{C} 11-\mathrm{O} 1$ & $56.2(3)$ \\
\hline $\mathrm{N} 4-\mathrm{N} 3-\mathrm{C} 1-\mathrm{N} 5$ & $72.9(3)$ & $\mathrm{N} 3-\mathrm{C} 1-\mathrm{C} 11-\mathrm{O} 1$ & $-64.8(3)$ \\
\hline $\mathrm{C} 7-\mathrm{N} 3-\mathrm{C} 1-\mathrm{N} 5$ & $-100.4(3)$ & & \\
\hline
\end{tabular}

Hydrogen-bond geometry $\left(\AA,{ }^{\circ}\right)$

\begin{tabular}{lllll}
\hline$D-\mathrm{H} \cdots A$ & $D-\mathrm{H}$ & $\mathrm{H} \cdots A$ & $D \cdots A$ & $D-\mathrm{H} \cdots A$ \\
\hline $\mathrm{C} 5-\mathrm{H} 5 A \cdots \mathrm{N} 2^{\mathrm{i}}$ & 0.95 & 2.51 & $3.453(4)$ & 171 \\
$\mathrm{C} 9-\mathrm{H} 9 A \cdots \mathrm{N} 2^{\mathrm{ii}}$ & 0.95 & 2.61 & $3.433(4)$ & 145 \\
$\mathrm{C} 4-\mathrm{H} 4 A \cdots \mathrm{O} 1^{\mathrm{iii}}$ & 0.95 & 2.53 & $3.444(4)$ & 162 \\
\hline
\end{tabular}

Symmetry codes: (i) $x,-y+1, z+1 / 2$; (ii) $x, y, z+1$; (iii) $x+1 / 2,-y+1 / 2, z+1 / 2$. 